\title{
Topologically Stable States of the Anti-Centrifugal Potential
}

\author{
Rossen Dandoloff \\ Laboratoire de Physique Theorique et Modelisation, Universite de Cergy-Pontoise, Cergy-Pontoise, France \\ Email: rdandoloff@yahoo.com
}

How to cite this paper: Dandoloff, R. (2019) Topologically Stable States of the Anti-Centrifugal Potential. Journal of Modern Physics, 10, 1002-1005. https://doi.org/10.4236/jmp.2019.108066

Received: June 9, 2019

Accepted: July 16, 2019

Published: July 19, 2019

Copyright $\odot 2019$ by author(s) and Scientific Research Publishing Inc. This work is licensed under the Creative Commons Attribution International License (CC BY 4.0).

http://creativecommons.org/licenses/by/4.0/

\begin{abstract}
We present a study of the anti-centrifugal potential based on the incorporation of the quantum geometric potential of a surface [1] into the generalised anti-centrifugal potential [2]. As a basic variable we will use the unit normal to the surface. Then the total quantum effective potential appears to be the nonlinear sigma model plus positive terms. A $2 \mathrm{~d}$ bilayer geometry smoothly connected by a neck is used to show that the anti-centrifugal potential creates topologically stable states.
\end{abstract}

\section{Keywords}

Anti Centrifugal Potential, Topology, Quantum States

\section{Introduction}

Recently a peculiar quantum behaviour of a free particle on a plane $\left(R^{2}\right)$ was discussed [3], where free particles with 0 angular momentum $(m=0)$ appear to be attracted to the origin of the plane (the origin of the coordinate system on the plane). The authors [3] have considered a Dirac $\delta$-function potential at the origin, which breaks the translational invariance of the plane and introduces a length scale in the problem. It turns out that a Hamiltonian with a Dirac $\delta$-function potential on the plane is equivalent to a free Hamiltonian on a plane with one point (at the origin) taken out [4]. Then the plane becomes non simply connected and the consequences for quantum mechanics are studied in [4]: namely the self adjointness of operators. In the present paper we will discuss a non simply connected surface and will study the consequences of this topological property of the surface for the stability of the anti centrifugal states. We will follow our previous work [2] on the generalised anti centrifugal potential. In this paper [2] we have analysed the Laplace-Beltrami (LBO) operator on a surface where we have introduced half-geodesic coordinates [5]. The radial coordinate 
$\xi$ represents the geodesic and the other $\eta$ closes around the hole at the origin. The line element in these coordinates is given by: $\mathrm{d} s^{2}=\mathrm{d} \xi^{2}+h(\xi) \mathrm{d} \eta^{2}$. The LBO separates in two parts: kinetic and potential part $V$ for the radial coordinate $\xi$ given by

$$
V=-\frac{\hbar^{2}}{2 m_{0}}\left[\frac{1}{4} k_{g}^{2}-\frac{1}{2} K-\frac{m^{2}}{r_{0}^{2}}\right]
$$

Here $K=-h^{-1} \partial_{\xi}^{2} h$ represents the Gaussian curvature of the surface and $k_{g}=-h^{-1} \partial_{\xi} h$ is the geodesic curvature. For angular momentum $m=0$ (note that the anti centrifugal potential appears only for $m=0$ ) the above potential takes the form:

$$
V=-\frac{\hbar^{2}}{2 m_{0}}\left[\frac{1}{4} k_{g}^{2}-\frac{1}{2} K\right]
$$

In addition for a general surface there is a quantum geometric potential (QGP). Usually it is shown that this potential is induced on a surface, using the method of a very high sandwich potential around the surface. This potential has the form [1]

$$
V=-\frac{\hbar^{2}}{8 m_{0}}\left(\kappa_{1}-\kappa_{2}\right)^{2}=-\frac{\hbar^{2}}{2 m_{0}}\left(M^{2}-K\right) .
$$

Here $\kappa_{i}$ 's are the principal curvatures of the surface, $\hbar$ is the Planck's constant and $m_{0}$ is the effective mass. $M$ is the Mean curvature and $K$ is the Gauss curvature of a two-dimensional surface embedded in three-dimensional space.

The combined potential $V_{\text {tot }}$ reads:

$$
V_{t o t}=-\frac{\hbar^{2}}{2 m_{0}}\left[\frac{1}{4} k_{g}^{2}-\frac{3}{2} K+M^{2}\right]
$$

\section{The Normal n of a Surface as a Main Variable}

The investigations until now ware concentrated on different geometries and the corresponding QGP. So far the link between the QGP and the topology of the underlying geometry has not been discussed.

In this paper we will use the normal to the surface $\mathbf{n}$ as the basic variable describing the surface. Indeed the link between the normal $\mathbf{n}$ of a surface and its Gaussian and Mean curvatures is given by the following expressions [6]:

$$
\begin{gathered}
M=-\nabla \cdot \mathbf{n}, \\
2 K=\mathbf{n} \cdot \nabla^{2} \mathbf{n}+(\nabla \cdot \mathbf{n})^{2} .
\end{gathered}
$$

Now we are ready to express $V_{\text {tot }}$ in terms of the normal $\mathbf{n}$ only.

$$
V_{\text {tot }}=-\frac{\hbar^{2}}{2 m_{0}}\left[\frac{1}{4} k_{g}^{2}+\frac{1}{4}(\nabla \cdot \mathbf{n})^{2}+\frac{3}{4}(\nabla \mathbf{n})^{2}\right]
$$

Here we have used the following identity:

$$
\mathbf{n}^{2}=1 \Rightarrow \mathbf{n} \cdot \nabla^{2} \mathbf{n}=-(\nabla \mathbf{n})^{2}
$$


Now we will consider here a surface that represents a double layer connected by a smooth neck. The normals $\mathbf{n}$ to this surface at the infinity on the upper layer are directed upwards and on the lower layer are directed downwards. Let us note that the catenoid posses the same topology as the above described double layer. Usually the anti centrifugal potential is considered in cylindrical coordinates because there its presence becomes immediately evident. In this case: $V_{\text {eff }}=V(r)+\frac{m^{2}}{2 m_{0} r^{2}}$ where $r$ is the radial coordinate-in our case this is $\xi$.

\section{The Number of States}

Now we would like to estimate the number of radial states (for the effective one dimensional Schrödinger equation in the $\xi$ direction). The momenta $p_{\xi}$ in the potential $V_{\xi}=V_{\text {tot }}$ vary between 0 and $p_{\xi}$ where

$$
p_{\xi}=\sqrt{-2 m_{0} V_{\xi}}=\frac{\hbar}{2} \sqrt{(\nabla \cdot \vec{n})^{2}+3(\nabla \vec{n})^{2}+k_{g}^{2}} \geq \frac{\hbar}{2} \sqrt{3(\nabla \vec{n})^{2}} .
$$

In the inequality we have used the fact that $k_{g}^{2}$ and $(\nabla \cdot \vec{n})^{2}$ are always positive. When $\xi \rightarrow \infty \quad \tilde{\mathbf{n}} \rightarrow \tilde{\mathbf{n}}_{0}=$ constant vector field, therefore $V_{\xi} \rightarrow 0$ (note that $k_{g} \rightarrow 0$ for $\xi \rightarrow \infty$ and that the potential $V(\xi)$ represents a smooth function of $\xi$ because we assume that the surface is smooth surface and as a consequence $\overrightarrow{\mathbf{n}}$ is a smooth function of $\xi$ ) and $p_{\xi}=0$ for $\xi=\infty$, so $\xi=\infty$ represents a turning point for the quasi-classical approximation in the framework of which we are estimating the number of localised states. Now we are ready to evaluate the density of states along the $\xi$ axis. The density of states is given by the following expression [7]:

$$
\mathrm{d} \mathcal{N}=\frac{p_{\xi} \mathrm{d} \xi}{2 \pi \hbar}=\frac{p_{\xi} \mathrm{d} \xi}{h} \geq \frac{\sqrt{3}}{4 \pi}|\nabla \vec{n}| \mathrm{d} \xi=\frac{\sqrt{3}}{4 \pi} \mathrm{d} \theta .
$$

where $\theta(\xi)$ is the azymutal angle in the $\xi$ direction. The unit vector that represents the normal to the surface is given by $\vec{n}=(\cos \theta(\xi), \sin \theta(\xi))$. Note that usually in the context of different spin models $\left(|\nabla \vec{n}|^{2} \mathrm{~d} \xi\right)$ represents the energy density and is the one dimensional non linear sigma model. Then the total number of states is:

$$
N=\oint \mathrm{d} \mathcal{N}=\oint \frac{\sqrt{3}}{4 \pi} \mathrm{d} \theta=\frac{\sqrt{3}}{2}>0 .
$$

The integration path represents the equivalence of two disconnected half circles to the left of the origin and to the right of the origin of the coordinate system. The total gives $2 \pi(\oint \mathrm{d} \theta=2 \pi)$.

\section{Conclusion}

It is clear that using topological arguments we cannot determine the exact number of states. But on the other hand the localised states cannot be destroyed by any smooth deformation of the considered surface i.e. they are topologically stable. These topological considerations give the localised anti-centrifugal states addi- 
tional legitimacy.

\section{Conflicts of Interest}

The author declares no conflicts of interest regarding the publication of this paper.

\section{References}

[1] da Costa, R.C.T. (1981) Physical Review A, 23, 1982. https://doi.org/10.1103/PhysRevA.23.1982

[2] Dandoloff, R., Jensen, B. and Saxena, A. (2014) Physics Letters A, 378, 510. https://doi.org/10.1016/j.physleta.2013.12.016

[3] Cirone, M.A., Rzazewski, K., Schleich, W.P., Straub, F. and Wheeler, J.A. (2001) Physical Review A, 65, Article ID: 022101. https://doi.org/10.1103/PhysRevA.65.022101

[4] Kowalski, K., Podlaski, K. and Rembielinski, J. (2002) Physical Review A, 66, Article ID: 032118. https://doi.org/10.1103/PhysRevA.66.032118

[5] Forsyth, A.R. (2012) Lectures on the Differential Geometry of Curves and Surfaces. Forgotten Books, London.

[6] Weatherburn, C.E. (1961) Differential Geometry of Three Dimensions. Vol. 1, Cambridge University Press, Cambridge.

[7] Landau, L.D. and Lifshitz, E.M. (2004) Quantum Mechanics. Butterworth-Heinemann, Oxford. 